\title{
Correlation of Tc-99 m ethyl cysteinate dimer single-photon emission computed tomography and clinical presentations in patients with low cobalamin status
}

Min-Chien $\mathrm{Tu}^{1,4^{*}}$, Chung-Ping Lo ${ }^{2,4}$, Ching-Yuan Chen ${ }^{3,4}$ and Ching-Feng Huang ${ }^{1,4}$

\begin{abstract}
Background: Cobalamin (Cbl) deficiency has been associated with various neuropsychiatric symptoms of different severities. While some studies dedicated in structural neuroimaging credibly address negative impact of low Cbl status, functional imaging reports are limited. We herein retrospectively review the correlation of Tc-99 m ethyl cysteinate dimer single-photon emission computed tomography (Tc-99 m-ECD SPECT) and clinical presentations among patients with low serum cobalamin (Cbl) status $(<250 \mathrm{pg} / \mathrm{ml})$.
\end{abstract}

Methods: Twelve symptomatic patients with low serum Cbl status were enrolled. Clinical presentations, Tc-99 $m-E C D$ SPECT, and neuropsychological tests were reviewed.

Results: Dysexecutive syndrome (67\%), forgetfulness (50\%), attention deficits (42\%), and sleep disorders (33\%) constituted the major clinical presentations. All patients (100\%) had temporal hypoperfusion on the Tc-99 m-ECD SPECT. Five patients (42\%) had hypoperfusion restricted within temporal regions and deep nuclei; seven patients (58\%) had additional frontal hypoperfusion. In patients with hypoperfusion restricted within temporal regions and deep nuclei, psychiatric symptoms with spared cognition were their main presentations. Among patients with additional frontal hypoperfusion, six of seven patients ( $86 \%$ ) showed impaired cognitive performances (two of them were diagnosed as having dementia). Among ten patients who finished neuropsychological tests, abstract thinking (70 \%) was the most commonly affected, followed by verbal fluency (60\%), short-term memory (50\%), and attention (50\%). Anxiety and sleep problems were the major clinically remarkable psychiatric features (33\% both). Four Tc-99 $\mathrm{m}$-ECD SPECT follow-up studies were available; the degree and extent of signal reversal correlated with cognitive changes after $\mathrm{Cb}$ replacement therapy.

Conclusions: Our TC-99 m-ECD SPECT observations provide pivotal information of neurobiological changes within basal ganglia and fronto-temporal regions in conjunction with disease severity among patients with Cbl deficiency. Hypoperfusion within thalamus/basal ganglia and temporal regions may be seen in the earlier state of Cbl deficiency, when psychiatric symptoms predominate. Hypoperfusion beyond thalamus/basal ganglia and involving frontal regions appears when cognitive problems, mostly dysexecutive syndrome, are manifested. Symmetric hypofrontality of SPECT in the context of dysexcutive syndrome serves as a distinguishing feature of non-amnestic mild cognitive impairment attributed to Cbl deficiency. Concordant with TC-99 m-ECD SPECT findings, the psychiatric symptoms and dysexcutive syndrome undergird impaired limbic and dorsolateral prefrontal circuits originating from basal ganglia respectively.

Keywords: Cobalamin deficiency, Vitamin B12, Tc-99 m-ECD, SPECT, Cognition, Mild cognitive impairment

\footnotetext{
* Correspondence: tmctmc30@yahoo.com.tw

'Department of Neurology, Taichung Tzu Chi Hospital, Buddhist Tzu Chi

Medical Foundation, Taichung, Taiwan

${ }^{4}$ School of Medicine, Tzu Chi University, Hualien, Taiwan

Full list of author information is available at the end of the article
}

\section{Ciomed Central}

(c) 2015 Tu et al. Open Access This article is distributed under the terms of the Creative Commons Attribution 4.0 International License (http://creativecommons.org/licenses/by/4.0/), which permits unrestricted use, distribution, and reproduction in any medium, provided you give appropriate credit to the original author(s) and the source, provide a link to the Creative Commons license, and indicate if changes were made. The Creative Commons Public Domain Dedication waiver (http:// creativecommons.org/publicdomain/zero/1.0/) applies to the data made available in this article, unless otherwise stated. 


\section{Background}

Cobalamin (Cbl) deficiency has been associated with various neuropsychiatric symptoms of different severities [1]. Delayed detection of Cbl deficiency among the elderly is sometimes complicated with severe neurological consequences; hence determination of $\mathrm{Cbl}$ status is often included in the diagnostic workup for dementia [2]. Serology evaluations of $\mathrm{Cbl}$ level are also frequently enlisted as part of diagnostic repertoire for mild cognitive impairment (MCI), an increasingly-identified syndrome in which changes of cognition exist but not to the extent of dementia [3]. Although previous literatures have addressed the negative impact of low Cbl status on myelin formation, cellular membrane stability, and neurotransmitters regulation [4], the causality between cognition decline and $\mathrm{Cbl}$ status remains inconclusive [1]. As subtle neuropathological changes in the brain may precede profound symptoms or clinical dementia, neuroimaging study provides a useful tool to probe fundamental mechanisms of disease process. While some studies dedicated in structural neuroimaging credibly address negative impact of low Cbl status [5, 6], functional imaging reports are limited $[7,8]$. Therefore, we conducted a surveillance to integrate the clinical presentations and Tc-99 m ethyl cysteinate dimer single-photon emission computed tomography (Tc-99 m-ECD SPECT) among patients with low Cbl status.

\section{Methods}

We retrospectively reviewed twelve symptomatic patients with low serum $\mathrm{Cbl}$ status $(<250 \mathrm{pg} / \mathrm{ml})$ [9]. Their main symptoms were featured with cognitive (e.g., forgetfulness and difficulty on planning) and/or psychiatric (e.g., sleep problems and low mood) complaints. All patients had checked complete blood count, thyroid function, cortisol, serum folic acid levels, and rapid plasma reagin screen, which were within normal limits. All patients received brain magnetic resonance imaging (MRI) for excluding cerebral vascular occlusion and/or large cortical infarcts, which would be possible confounding factors to SPECT observations. Tc-99 m-ECD SPECT scan interpretations and clinical profiles, including demographic data and neuropsychological tests, were collected. The written informed consent was obtained from all participants and there relevant radiation exposure was under regulation of radiometry in our country. The study was permitted by the Institutional Review Board of the Taichung Tzu Chi Hospital, Buddhist Tzu Chi Medical Foundation (REC 103-47).

\section{Tc-99 m-ECD SPECT}

Twelve patients underwent a Tc-99 m-ECD SPECT scan at rest, conducted before or one week within the start of Cbl supplement. A brain scintigraphy was performed
$30 \mathrm{~min}$ after intraveneous injection of $740 \mathrm{Mbq} 99 \mathrm{mTc}-$ ECD on General Electric, Infinia Hawkeye 4. Subsequently, a combined SPECT equipped with a dual-head gamma-camera with low-energy high resolution collimators, a $7 \%$ energy window centered at $140 \mathrm{keV}$ and $128 \times$ 128 matrix (GE, USA) was conducted. Acquisition was performed in continuous mode with 240 projections in a $128 \times 128$ matrix, zoom: 1 . The camera operated in segmented mode with double $360 * 2{ }^{\circ}$ rotation with $3{ }^{\circ}$ per projection angle. No scatter windows were placed. Through the mathematical approximation technique, acquired data were reconstructed (ordered-subset expectation maximization attenuation scatter method; Chang order 0; threshold 10; coefficient 0.11; cutoff frequency, 0.3 cycles/pixel for the main) after applying a Butterworth filter. The SPECT was displayed on GE Xeleris 2 workstation in axial, sagittal, and coronal slices. A count difference cost function with an iterative downhill-simplex search algorithm was applied for registration. The images were displayed on a computer monitor using an identical cool color scale (window, 100; base, 0 ).

Both visual and quantitative image analyses were performed. For visual analysis, images were reported independently by two nuclear medicine physicians who were blind to radiological and clinical results. Should there be any discrepancy between the interpretations, a consensus would be reached after panel discussion. For the quantitative analysis, 4 fixed axial images with predefined templates were selected (Fig. 1). Ten regions of interest, including six in bilateral cerebral cortices (frontal, temporal, and parietal regions) and four in deep nuclei (thalamus and basal ganglia), were symmetrically analyzed in each hemisphere. The regions of interest ranged in pixels from 90 (deep nuclei) to 170 (cerebral cortices) and were fixed in size across studies. All regions of interest were placed by the same operator to eliminate interoperator variability. The intraoperator reliability in the quantitative measurements was found to be Kappa $=0.821(p<0.001), 95 \%$ CI $(0.602,1.040)$ ( $n=12$ times). The mean counts in each selected region were normalized with respect to the mean counts in the ipsilateral cerebellum to quantify regional ECD uptake. In order to represent regional signal changes and avoid selection bias, final ECD uptake would be calculated by averaging data in which templates were placed onto three sequential neighboring slices. The average values of regions of interest to cerebellum ratio less than 0.8 were defined as abnormal and recorded [10].

\section{Neuropsychological tests}

Neuropsychological tests were available in ten patients. Mini-mental state examination (MMSE) [11], Cognitive Abilities Screening Instrument (CASI) [12], Clinical Dementia Rating (CDR) [13], and neuropsychiatric 

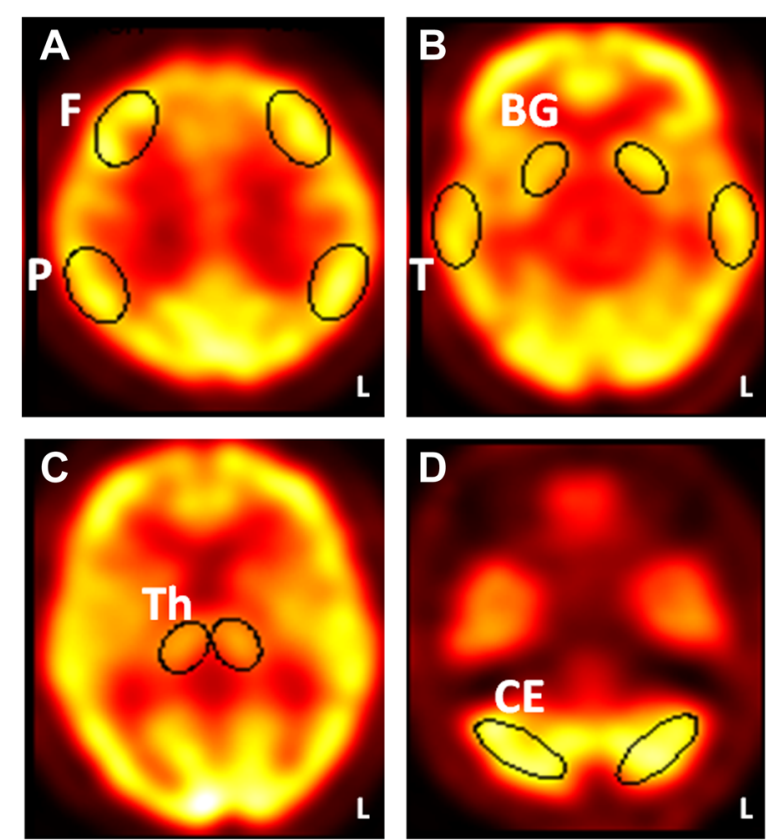

Fig. 1 Regions-of-interest templates placed on selected axial images of single-photon emission computed tomography (SPECT). (a) $F=$ frontal regions; $\mathrm{P}=$ parietal regions. (b) $\mathrm{T}=$ temporal regions; $\mathrm{BG}=$ basal ganglia. (c) $\mathrm{Th}=$ thalamus. (d) $\mathrm{CE}=$ cerebellum

inventory (NPI) [14] were thoroughly reviewed. In both MMSE and CASI, higher score represents better cognitive achievement. A MMSE score less than 26 would conventionally be classified as indicative of cognitive impairment [11]. CASI total scores below the cut-off score of normative data in Taiwan were registered as abnormal [15]. Additionally, CASI provides quantitative assessment of 9 cognitive subdomains, including long term memory, short term memory, attention, mental manipulation, orientation, abstract thinking, language abilities, drawing, and verbal fluency. Scores of CASI subdomain falling more than one standard deviation of normative data in Taiwan were registered as abnormal [15]. CDR was applied to evaluate the daily functional performance related with the severity of cognitive deficits $(0=$ Normal; $0.5=$ Very mild; $1=$ Mild; $2=$ Moderate; $3=$ Severe). NPI, in which twelve psychological symptoms were determined, were registered clinically-remarkable if score of each defined symptom more than 4 [14].

\section{Results}

\section{Tc-99 m-ECD SPECT}

Tc-99 m-ECD SPECT findings in line with clinical profiles were summarized in Table 1. The patients' age ranged from 29 to 81 . Dysexecutive syndrome (67\%), forgetfulness (50\%), attention deficits (42\%), and sleep disorders (33\%) constituted the major clinical presentations. All patients $(100 \%)$ had temporal hypoperfursion on the Tc-
99 m-ECD SPECT. Five patients (42 \%) (Patient \#8 \#12) had hypoperfusion restricted within temporal regions and deep nuclei; seven patients (58\%) (Patient \#1 \#7) had frontal hypoperfusion in addition to temporal hypoperfusion. In patients with hypoperfusion restricted within temporal regions and deep nuclei, their cognitive performances were intact (MMSE $=26 \sim 27$; CASI $=75 \sim 93)$ and daily functional performance were relatively preserved $(\mathrm{CDR}=0 \sim 0.5)$. Three of five patients $(60 \%)$ presented with psychiatric symptoms (i.e., irritability, anxiety, and sleep disorders). Among patients with additional frontal hypoperfusion, six of seven patients (86\%) showed impaired cognitive performances (MMSE $=12 \sim 25 ;$ CASI $=34 \sim 82$ ) and impaired daily functional performance $(\mathrm{CDR}=$ $0.5 \sim 1)$. Only one patient had normal achievement of neuropsychological tests and intact daily functional status (Patient \#7). Dysexecutive syndrome (86 \%) and attention deficits (57\%) constituted the major clinical presentations. Anxiety and insomnia were recorded in three patients (43\%). Two patients (29\%) were eventually diagnosed with dementia.

Quantitative assessment of Tc-99 m-ECD SPECT was detailed in Table 2. Data were presented as individual value of three consecutive slices and their average. From the data of cerebral cortices, abnormal ratio counts within temporal regions were identified in all patients (eleven as bilateral and one as unilateral involvement). Abnormal ratio counts within frontal regions were identified in seven patients $(\mathrm{Pa}-$ tient \#1-\#7); all presented as bilateral involvement. From the data of deep nuclei, ten had impaired ratio count within thalamus (eight as bilateral and two as unilateral involvement). Five had impaired ratio count within basal ganglia.

Intriguingly, Tc-99 m-ECD SPECT changed in accordance with different clinical severity. In Patients \#11 and \#12, whose CDR staged 0 and ages were younger, had neuropsychiatric symptoms and hypoperfusion within temporal regions on Tc-99 m-ECD SPECT. Among cases whose CDR staged 0.5 or greater, hypoperfusions of dorsolateral prefrontal regions turned to be prominent as dysexcutive symptoms and attention deficits prevailed (Fig. 2).

\section{Neuropsychological tests}

Ten patients who received complete neuropsychological tests (Patient \#1 \#10) were further analyzed. Abstract thinking (70 \%) was the cognitive domains most commonly affected, followed by verbal fluency (60\%), shortterm memory (50\%), and attention (50\%) (Fig. 3). In NPI evaluations, there were three patients identified as having clinically remarkable anxiety and sleep problems (30 \%). Only one patient had clinically remarkable 
Table 1 Demographic data and single-photon emission computed tomography observation of patients with cobalamin deficiency

\begin{tabular}{|c|c|c|c|c|c|c|c|c|}
\hline $\begin{array}{l}\text { Patient } \\
\#\end{array}$ & $\begin{array}{l}\text { Age/ } \\
\text { Gender }\end{array}$ & $\begin{array}{l}\text { Education } \\
\text { level (years) }\end{array}$ & $\begin{array}{l}\text { Serum cobalamin } \\
\text { level }(\mathrm{pg} / \mathrm{ml})\end{array}$ & $\begin{array}{l}\text { Clinical } \\
\text { Dementia } \\
\text { Rating }\end{array}$ & $\begin{array}{l}\text { Mini-Mental } \\
\text { State } \\
\text { Examination }\end{array}$ & $\begin{array}{l}\text { Cognitive Abilities } \\
\text { Screening Instrument }\end{array}$ & $\begin{array}{l}\text { Single-photon emission } \\
\text { computed tomography }\end{array}$ & Clinical presentations \\
\hline 1 & $73 / F$ & 0 & 199 & 1 & 12 & 34 & $\begin{array}{l}\text { Bil. F. T. P. Tha. } \\
\text { (more severe in Lt) } \\
\text { Lt BG. }\end{array}$ & $\begin{array}{l}\text { Forgetfulness, } \\
\text { attention deficits, } \\
\text { dysexcutive } \\
\text { syndrome, and } \\
\text { dementia }\end{array}$ \\
\hline 2 & $80 / F$ & 0 & 230 & 1 & 13 & 42 & Bil. F. T. Tha. Rt BG & $\begin{array}{l}\text { Forgetfulness } \\
\text { and dementia }\end{array}$ \\
\hline 3 & $60 / F$ & 6 & 207 & 0.5 & 24 & 78 & Bil. F. T. Tha. & $\begin{array}{l}\text { Insomnia, forgetfulness, } \\
\text { attention deficits, and } \\
\text { dysexcutive syndrome }\end{array}$ \\
\hline 4 & $81 / \mathrm{M}$ & 12 & 177 & 0.5 & 25 & 82 & $\begin{array}{l}\text { Bil. F. T. Tha.BG } \\
\text { (more severe in Rt) }\end{array}$ & $\begin{array}{l}\text { Depression, } \\
\text { forgetfulness, attention } \\
\text { deficits, and } \\
\text { dysexcutive syndrome }\end{array}$ \\
\hline 5 & $53 / F$ & 6 & 218 & 0.5 & 22 & 65 & Bil. F. T. Tha. & $\begin{array}{l}\text { Dysexecutive } \\
\text { syndrome, anxiety, and } \\
\text { insomnia }\end{array}$ \\
\hline 6 & $74 / F$ & 6 & 209 & 0.5 & 21 & 61 & Bil. F. T. Tha. & $\begin{array}{l}\text { Forgetfulness, } \\
\text { attention deficits, } \\
\text { dysexcutive syndrome, } \\
\text { and anxiety }\end{array}$ \\
\hline 7 & $58 / F$ & 12 & 210 & 0 & 28 & 88 & Bil. F. T. Tha. & $\begin{array}{l}\text { Anxiety, insomnia, and } \\
\text { dysexcutive syndrome }\end{array}$ \\
\hline 8 & $56 / F$ & 12 & 229 & 0.5 & 27 & 87 & Bil. T. Tha. & $\begin{array}{l}\text { Dysexecutive } \\
\text { syndrome }\end{array}$ \\
\hline 9 & $65 / F$ & 6 & 202 & 0.5 & 26 & 93 & Bil. T. Rt Tha. & Attention deficits \\
\hline 10 & $61 / M$ & 6 & 108 & 0.5 & 27 & 75 & $\begin{array}{l}\text { Bil. T. (more severe in Lt) } \\
\text { Lt BG }\end{array}$ & $\begin{array}{l}\text { Irritability, } \\
\text { forgetfulness, and } \\
\text { dysexcutive syndrome }\end{array}$ \\
\hline 11 & $29 / F$ & 12 & 199 & 0 & N.A. & N.A. & Lt T. Tha. & $\begin{array}{l}\text { Irritability, anxiety, } \\
\text { dysthymia, and sleep } \\
\text { disorders }\end{array}$ \\
\hline 12 & $41 / \mathrm{M}$ & 12 & 175 & 0 & N.A. & N.A & Bil. T. Rt BG & Anxiety \\
\hline
\end{tabular}

Abbreviations: $F$ Famale, M: Male, N.A non-available, Bil bilateral, $R t$ right, $L t$ left, $F$ frontal region, $T$ temporal region, $P$ parietal region, Tha thalamus, $B G$ basal ganglia

depression (10\%). Six patients were re-examined neuropsychological tests 3 months later after $\mathrm{Cbl}$ replacement therapy, when normalization of serum $\mathrm{Cbl}$ level was achieved. Three of them had improvement (Patient \#3: 24 to 28; Patient \#7: 28 to 30; Patient \#8: 27 to 29) but the other three (Patient \#1: 12 to 11; Patient \#6: 21 to 18; Patient \#10: 27 to 23) had decline of MMSE total score.

\section{Tc-99 m-ECD SPECT follow-up studies}

Tc-99 m-ECD SPECT follow-up studies were available in four patients. They were proceeded three to five months after Cbl replacement therapy (Patient \#1: 5 months; Patient \#3: 5 months; Patient \#7: 5 months; Patient \#8: 3 months). In visual assessment, signals reversal was identified within most regions that were originally impaired, the extent and degree of signal reversal correlated with individual therapeutic responses (Fig. 4).

In quantitative assessment of cerebral cortices (Table 3), Patient \#3, \#5, \#7 had global post-treatment increase over baseline value compared with Patient $\# 1$, who had increased value limited within right fronto-temporal and bilateral parietal regions. Patient \#3, \#5, \#7 also had more intense post-treatment increase within fronto-temporal regions than Patient \#1. In the assessment of deep nuclei, although all patients had signal increase, only Patient \#7 and \#8 had signal normalization.

\section{Discussion}

The aim of this work is to elucidate TC-99 m-ECD SPECT findings in conjunction with clinical presentations and symptom severity among patients with $\mathrm{Cbl}$ deficiency. The selective involvement at bilateral fronto-temporal regions 
Table 2 Regions-to-cerebellar ratio among patients with cobalamin deficiency

\begin{tabular}{|c|c|c|c|c|c|c|c|c|c|c|c|c|c|c|c|c|c|c|c|c|c|c|c|c|c|c|c|c|c|c|}
\hline & \multicolumn{6}{|c|}{ Frontal regions } & \multicolumn{6}{|c|}{ Temporal regions } & \multicolumn{6}{|c|}{ Parietal regions } & \multicolumn{6}{|c|}{ Thalamus } & \multicolumn{6}{|c|}{ Basal ganglia } \\
\hline & \multicolumn{3}{|l|}{$\mathrm{Rt}$} & \multicolumn{3}{|l|}{$\mathrm{Lt}$} & \multicolumn{3}{|l|}{$\overline{\mathrm{Rt}}$} & \multicolumn{3}{|l|}{$\mathrm{Lt}$} & \multicolumn{3}{|l|}{ Rt } & \multicolumn{3}{|l|}{$\mathrm{Lt}$} & \multicolumn{3}{|l|}{$\mathrm{Rt}$} & \multicolumn{3}{|l|}{$\mathrm{Lt}$} & \multicolumn{3}{|l|}{ Rt } & \multicolumn{3}{|l|}{$\mathrm{Lt}$} \\
\hline \multicolumn{31}{|c|}{ Patient \# } \\
\hline \multirow[t]{2}{*}{1} & .81 & .77 & .78 & .82 & .80 & .76 & .80 & .78 & .79 & .75 & .75 & .71 & .79 & .78 & .79 & .79 & .79 & .80 & .76 & .75 & .79 & .72 & .73 & .68 & .82 & .82 & .81 & .74 & .74 & .74 \\
\hline & & .79 & & & .79 & & & .79 & & & .74 & & & .79 & & & .79 & & & .77 & & & .71 & & & .82 & & & .74 & \\
\hline \multirow[t]{2}{*}{2} & .78 & .80 & .80 & .74 & .74 & .75 & .79 & .81 & .78 & .73 & .74 & .76 & .95 & .92 & .95 & .82 & .82 & .80 & .72 & .72 & .71 & .70 & .71 & .72 & .72 & .70 & .71 & .80 & .81 & .81 \\
\hline & & .79 & & & .74 & & & .79 & & & .74 & & & .94 & & & .81 & & & .72 & & & .71 & & & .71 & & & .81 & \\
\hline \multirow[t]{2}{*}{3} & .76 & .80 & .81 & .80 & .80 & .76 & .75 & .75 & .76 & .76 & .78 & .81 & .99 & 1.01 & .99 & .95 & .95 & .99 & .73 & .72 & .73 & .68 & .68 & .68 & .96 & .95 & .96 & .92 & .93 & .93 \\
\hline & & .79 & & & .79 & & & .75 & & & .78 & & & 1.00 & & & .96 & & & .73 & & & .68 & & & .96 & & & .93 & \\
\hline \multirow[t]{2}{*}{4} & .68 & .67 & .72 & .78 & .77 & .77 & .76 & .75 & .76 & .77 & .78 & .78 & .89 & .87 & .85 & .92 & .95 & .94 & .73 & .76 & .76 & .78 & .78 & .78 & .61 & .63 & .67 & .78 & .77 & .74 \\
\hline & & .72 & & & .77 & & & .76 & & & .78 & & & .87 & & & .94 & & & .75 & & & .78 & & & .64 & & & .76 & \\
\hline 5 & .76 & .76 & .80 & .73 & .80 & .77 & .75 & .75 & .71 & .77 & .76 & .76 & .82 & .82 & .86 & .83 & .82 & .83 & .77 & .78 & .78 & .67 & .66 & .72 & .83 & .84 & .83 & .82 & .82 & .82 \\
\hline & & .77 & & & .77 & & & .74 & & & .76 & & & .83 & & & .83 & & & .78 & & & .68 & & & .83 & & & .82 & \\
\hline 6 & .75 & .77 & .80 & .80 & .75 & .83 & .78 & .78 & .80 & .77 & .76 & .77 & .95 & .93 & .94 & .94 & .95 & .96 & .78 & .78 & .80 & .78 & .79 & .79 & .89 & .87 & .87 & .86 & .85 & .88 \\
\hline & & .77 & & & .79 & & & .79 & & & .77 & & & .94 & & & .95 & & & .79 & & & .79 & & & .88 & & & .86 & \\
\hline 7 & .78 & .80 & .76 & .79 & .77 & .77 & .75 & .75 & .74 & .75 & .75 & .73 & .85 & .87 & .85 & .84 & .81 & .83 & .67 & .69 & .69 & .69 & .69 & .70 & .91 & .92 & .94 & .93 & .95 & .93 \\
\hline & & .78 & & & .78 & & & .75 & & & .74 & & & .86 & & & .83 & & & .68 & & & .69 & & & .92 & & & .94 & \\
\hline 8 & .84 & .88 & .87 & .90 & .92 & .88 & .75 & .70 & .70 & .79 & .79 & .79 & .90 & .89 & .89 & .92 & .92 & .93 & .71 & .69 & .72 & .79 & .82 & .75 & .91 & .90 & .87 & .96 & .95 & .96 \\
\hline & & .86 & & & .90 & & & .72 & & & .79 & & & .89 & & & .92 & & & .71 & & & .79 & & & .89 & & & .96 & \\
\hline 9 & .86 & .87 & .85 & .97 & .92 & .94 & .77 & .77 & .77 & .80 & .79 & .79 & .82 & .83 & .82 & .97 & .94 & .97 & .66 & .65 & .64 & .83 & .83 & .83 & .94 & .92 & .92 & .96 & .99 & .95 \\
\hline & & .86 & & & .94 & & & .77 & & & .79 & & & .82 & & & .96 & & & .65 & & & .83 & & & .93 & & & .97 & \\
\hline 10 & .90 & .89 & .88 & .83 & .83 & .83 & .79 & .75 & .76 & .68 & .74 & .78 & .98 & .95 & .98 & .79 & .86 & .89 & .80 & .80 & .80 & .81 & .81 & .79 & .85 & .81 & .88 & .80 & .79 & .79 \\
\hline & & .89 & & & .83 & & & .77 & & & .73 & & & .97 & & & .85 & & & .80 & & & .80 & & & .85 & & & .79 & \\
\hline 11 & .93 & .92 & .86 & .83 & .88 & .90 & .82 & .82 & .81 & .72 & .72 & .72 & .98 & 1.02 & 1.00 & .95 & .85 & .91 & .86 & .84 & .80 & .77 & .72 & .74 & .98 & .99 & 1.00 & .95 & .93 & .95 \\
\hline & & .90 & & & .87 & & & .82 & & & .72 & & & 1.00 & & & .90 & & & .83 & & & .74 & & & .99 & & & .94 & \\
\hline 12 & .86 & .92 & .94 & .91 & .90 & .87 & .78 & .74 & .76 & .77 & .71 & .74 & .98 & .98 & .98 & .97 & .97 & .98 & .81 & .81 & .81 & .79 & .80 & .80 & .75 & .75 & .75 & .87 & .84 & .87 \\
\hline & & .91 & & & .89 & & & .76 & & & .74 & & & .98 & & & .97 & & & .81 & & & .80 & & & .75 & & & .86 & \\
\hline
\end{tabular}

Abbreviations: Rt right, $L t$ left; data were presented as ratio count from three consecutive slices (serif) followed by their average value (boldface) 

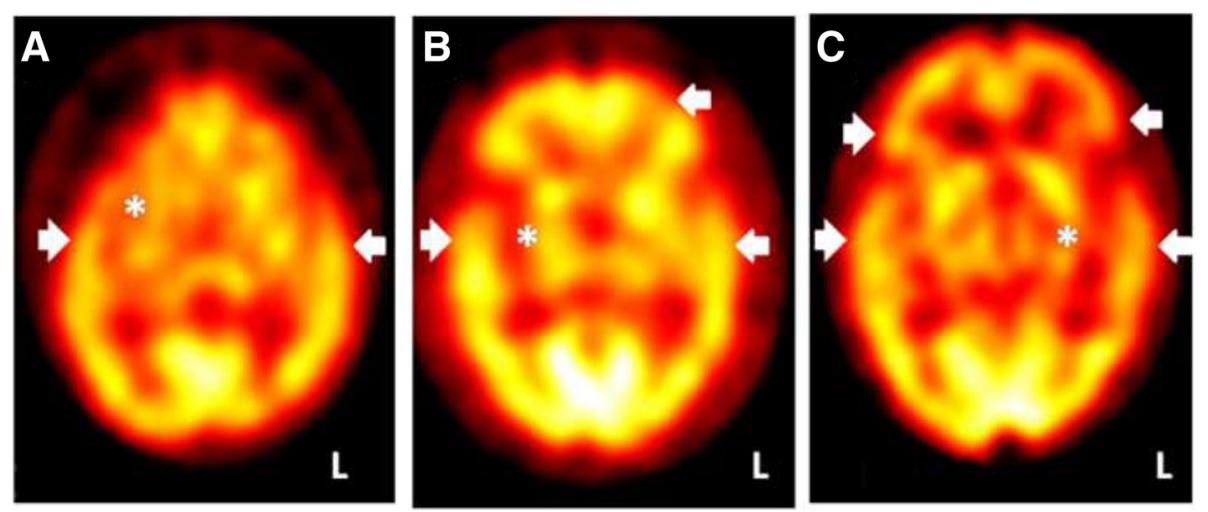

Fig. 2 Single-photon emission computed tomography (SPECT) correlates with clinical presentations and disease severity of cobalamin-deficient patients. (a) A case with anxiety disorder but preserved cognition. Clinical Dementia Rating (CDR) $=0$. SPECT shows hypoperfusion in right basal ganglia (asterisk) and bilateral temporal tips (arrow heads; Patient \#12). (b) A case with prominent dysexecutive syndrome. CDR $=0.5$. SPECT shows hypoperfusion in right basal ganglia (asterisk) and left dorsolateral prefrontal cortex, in addition to temporal regions. (arrow heads; Patient \#4). (c) A case with clinical diagnosis of dementia. CDR $=1$. SPECT shows bilateral, more pronounced in the left, thalamus (asterisk) and fronto-temporal regions hypoperfusion (arrow heads; Patient \#1)

seems to be the most consistent TC-99 m-ECD SPECT findings in Cbl-deficient patients. Prefrontal lesions commonly refer to executive dysfunction and vice versa [16]. Our cohort associates dysexecutive syndrome and attention deficits with hypofrontality of TC-99 m-ECD SPECT. It's the case that our patients with low serum $\mathrm{Cbl}$ status frequently encountered difficulty in planning personal agenda, managing situations of daily life, and tolerating changes of routine. There is a wealth of evidences suggesting roles of prefrontal regions in abstract thinking, verbal fluency, executive dysfunction, and attention maintenance $[17,18]$. The attention recruitment, highly allied with execution network, implement newly-generated schema and later assess their accuracy whenever an individual encounter a novel situation [19]. Moreover, it is hypothesized that the prefrontal cortex serves a specific function in cognitive control, in which patterns of activity that represent goals were actively maintained by shifting attention of all sensory modalities [20]. While prefrontal areas are pivotal for executive function and attention control, several other regions, including basal ganglia [18], thalamus, and cingulate gyrus are frequently recruited to accomplish implementation of such function [21]. The dorsolateral prefrontal circuits, as one of the major connections originating from the basal ganglia, govern implicit learning, sequencing, and allocation and filtering of attention [22]. These operations would be allocated in enhancing the efficiency of higher order processors such as execution, motor planning, and mental manipulation. Deactivation of basal ganglia would impair activity of frontal regions through dorsolateral prefrontal circuits, leading dysexecutive syndrome among Cbl-deficient patients.
Our TC-99 m-ECD SPECT also addresses the clinical importance of hypoperfusion within temporal lobes. While lateral and ventral portions of temporal lobe govern sensory input processing, medial portion of temporal lobe has been linked to encoding process of memory acquisition [23]. It is therefore reasonable to expect short-term memory deficits during the neuropsychological tests. Aside from the roles on cognition, there are prolific evidences of SPECT associating temporal lobe with various psychiatric symptoms [24]. The limbic circuits of basal ganglia, constituted by reciprocal projections between ventral part of striatum and temporal lobe, have also been approved of great impact on psychiatric disorders [22]. Two young patients in our studies (Patient \#11 and \#12), whose ages are far below commonly-known neurodegenerative diseases, address the negative impact of Cbl deficiency on cerebral metabolism. They often prone to worry about things that are not general cause for concern. From our cohort of patients with low Cbl status, anxiety and sleep problems are the major psychiatric features, and symptoms of anxiety and irritability might predate cognitive decline. By incorporating hypofunction of basal ganglia and fronto-temporal regions as the evidences of neurobiological abnormalities, distortion of cognition would further predispose anxiety and irritability, making patients with $\mathrm{Cbl}$ deficiency more vulnerable to react with changes of environmental stimuli.

We are convinced that TC-99 m-ECD SPECT evaluations not only provide neurobiological measures to undergird clinical phenomenology but serve as a supplementary tool for the workup of patients with cognitive decline. Frontal regions deactivation might serve as a surrogate 


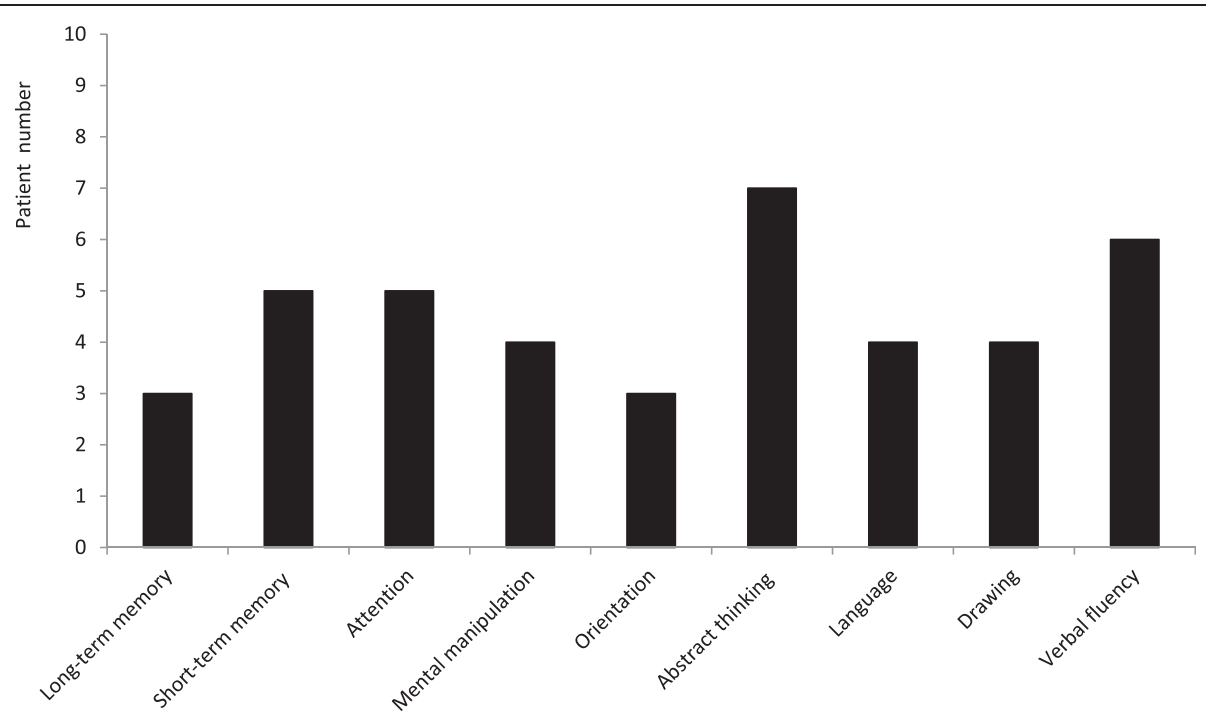

Fig. 3 Characterization of cognitive profiles of patients with low cobalamin status

marker for differentiating Cbl deficiency from Alzheimer's disease, in which radiouptake in precuneus, posterior cingulate gyrus, parietal and medial temporal lobes are preferentially impaired [24, 25]. Moreover, characterizing SPECT patterns would be of paramount clinical value before clinically-overt dementia occurs. Our study describes cognitive decline in a wide spectrum of severity, although the majority of our patients are categorized as $\mathrm{MCI}$ given with objective cognitive deficits but substantially preserved daily functional autonomy. Current researches frequently dichotomize MCI into amnestic and non-amnestic subtypes by neuropsychological tests. In contrast to the latter, amnestic subtype of MCI has greater possibility to share Alzheimer's disease pathology as it preludes demented
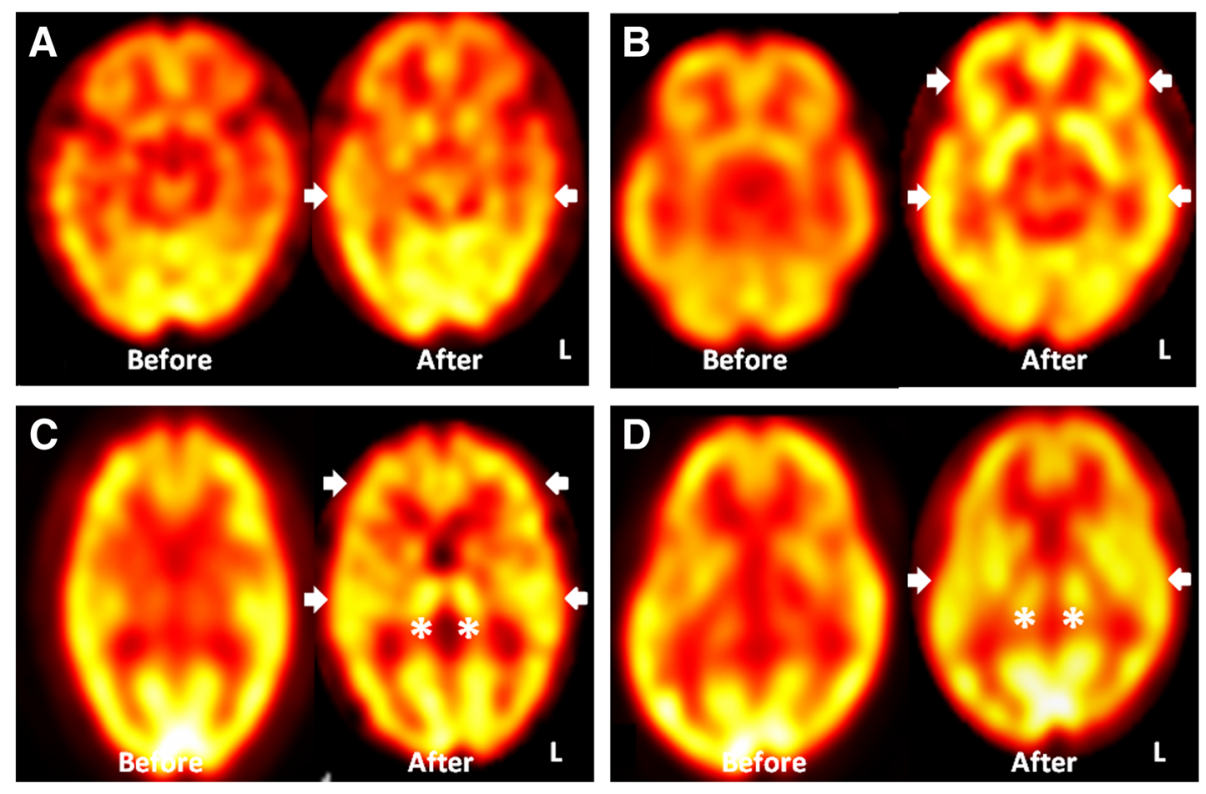

Fig. 4 Single-photon emission computed tomography (SPECT) signal changes in relation to cobalamin replacement therapy. (a) A demented patient with no improvement in cognition after therapy [Mini-Mental State Examination (MMSE) $=12$ to 11] had minimal signal regain only within temporal regions. (arrow heads; Patient \#1) (b) A mildly cognitive impaired patient with improvement in cognition after therapy (MMSE = 24 to 28) had avid SPECT signal recovery within bilateral fronto-temporal regions.(arrow heads; Patient \#3) (c) A patient with improvement of initial dysexecutive complaints and anxiety after therapy (MMSE = 28 to 30) had improving signals within bilateral thalamus (asterisk) and fronto-temporal regions.(arrow heads; Patient \#7) (d) A mildly cognitive impaired patient with improvement in cognition after therapy (MMSE $=27$ to 29$)$ had improving perfusion within bilateral thalamus (asterisk) and temporal regions from the follow-up SPECT. (arrow heads; Patient \#8) 
Table 3 Regions-to-cerebellar ratio changes in relation to cobalamin replacement therapy

\begin{tabular}{|c|c|c|c|c|c|c|c|c|c|c|c|c|c|c|c|c|c|c|c|c|c|c|c|c|c|c|c|c|c|c|}
\hline & \multicolumn{6}{|c|}{ Frontal regions } & \multicolumn{6}{|c|}{ Temporal regions } & \multicolumn{6}{|c|}{ Parietal regions } & \multicolumn{6}{|c|}{ Thalamus } & \multicolumn{6}{|c|}{ Basal ganglia } \\
\hline & \multicolumn{3}{|l|}{$\mathrm{Rt}$} & \multicolumn{3}{|l|}{$\mathrm{Lt}$} & \multicolumn{3}{|l|}{$\mathrm{Rt}$} & \multicolumn{3}{|l|}{$\mathrm{Lt}$} & \multicolumn{3}{|l|}{ Rt } & \multicolumn{3}{|l|}{$\mathrm{Lt}$} & \multicolumn{3}{|l|}{ Rt } & \multicolumn{3}{|l|}{$\mathrm{Lt}$} & \multicolumn{3}{|l|}{ Rt } & \multicolumn{3}{|l|}{$\mathrm{Lt}$} \\
\hline \multicolumn{31}{|l|}{ Patient\# } \\
\hline \multirow[t]{2}{*}{1 before } & .81 & .77 & .78 & .82 & .80 & .76 & .80 & .78 & .79 & .75 & .75 & .71 & .79 & .78 & .79 & .79 & .79 & .80 & .76 & .75 & .79 & .72 & .73 & .68 & .82 & .82 & .81 & .74 & .74 & .74 \\
\hline & & .79 & & & .79 & & & .79 & & & .74 & & & .79 & & & .79 & & & .77 & & & .71 & & & .82 & & & .74 & \\
\hline \multirow[t]{2}{*}{1 after } & .79 & .81 & .81 & .76 & .76 & .80 & .89 & .87 & .88 & .77 & .77 & .75 & 1.04 & 1.05 & 1.05 & .95 & .93 & .91 & .79 & .78 & .78 & .72 & .72 & .72 & .85 & .84 & .85 & .74 & .74 & .74 \\
\hline & & .80 & & & .77 & & & .88 & & & .76 & & & 1.05 & & & .93 & & & .78 & & & .72 & & & .85 & & & .74 & \\
\hline \multirow[t]{2}{*}{3 before } & .76 & .80 & .81 & .80 & .80 & .76 & .75 & .75 & .76 & .76 & .78 & .81 & .99 & 1.01 & .99 & .95 & .95 & .99 & .73 & .72 & .73 & .68 & .68 & .68 & .96 & .95 & .96 & .92 & .93 & .93 \\
\hline & & .79 & & & .79 & & & .75 & & & .78 & & & 1.00 & & & .96 & & & .73 & & & .68 & & & .96 & & & .93 & \\
\hline \multirow[t]{2}{*}{3 after } & .92 & .92 & .90 & .87 & .87 & .87 & .92 & .92 & .93 & .90 & .86 & .86 & 1.00 & 1.01 & 1.01 & .96 & .97 & .98 & .73 & .73 & .72 & .74 & .75 & .75 & .94 & .94 & .94 & .93 & .93 & .92 \\
\hline & & .91 & & & .87 & & & .92 & & & .87 & & & 1.01 & & & .97 & & & .73 & & & .75 & & & .94 & & & .93 & \\
\hline \multirow[t]{2}{*}{7 before } & .78 & .80 & .76 & .79 & .77 & .77 & .75 & .75 & .74 & .75 & .75 & .73 & .85 & .87 & .85 & .84 & .81 & .83 & .67 & .69 & .69 & .69 & .69 & .70 & .91 & .92 & .94 & .93 & .95 & .93 \\
\hline & & .78 & & & .78 & & & .75 & & & .74 & & & .86 & & & .83 & & & .68 & & & .69 & & & .92 & & & .94 & \\
\hline \multirow[t]{2}{*}{7 after } & .88 & .88 & .82 & .88 & .86 & .87 & .81 & .81 & .81 & .87 & .84 & .84 & .85 & .87 & .88 & .86 & .87 & .86 & .80 & .81 & .80 & .83 & .82 & .82 & .84 & .85 & .86 & .88 & .88 & .87 \\
\hline & & .86 & & & .86 & & & .81 & & & .85 & & & .87 & & & .86 & & & .80 & & & .82 & & & .85 & & & .88 & \\
\hline \multirow[t]{2}{*}{8 before } & .84 & .88 & .87 & .90 & .92 & .88 & .75 & .70 & .70 & .79 & .79 & .79 & .90 & .89 & .89 & .92 & .92 & .93 & .71 & .69 & .72 & .79 & .82 & .75 & .91 & .90 & .87 & .96 & .95 & .96 \\
\hline & & .86 & & & .90 & & & .72 & & & .79 & & & .89 & & & .92 & & & .71 & & & .79 & & & .89 & & & .96 & \\
\hline \multirow[t]{2}{*}{8 after } & .93 & .92 & .94 & .96 & .96 & .96 & 1.00 & 1.00 & 1.00 & 1.03 & 1.04 & 1.03 & .95 & .94 & .95 & 1.02 & 1.01 & .98 & .86 & .87 & .86 & .87 & .87 & .88 & .90 & .91 & .91 & .97 & .97 & .97 \\
\hline & & .92 & & & .96 & & & 1.00 & & & 1.03 & & & .95 & & & 1.00 & & & .86 & & & .87 & & & .91 & & & .97 & \\
\hline
\end{tabular}

Abbreviations: Rt right, $L t$ left; data were presented as ratio count from three consecutive slices (serif) followed by their average value (boldface) 
state in a closer temporal relationship [26]. The analysis of neuropsychological tests supports that non-amnestic subtype of MCI constituted as the majority of our cohort, whose TC-99 m-ECD SPECT features a pattern of symmetric hypoperfusion within frontotemporal regions. The widespread cerebral blood flow decrement not only stresses its relevance with metabolic derangement but also provides an interesting contrast to recent SPECT reports of $\mathrm{MCI}$, where asymmetric hypoperfusion over frontal regions and hippocampus occurs in association with nonamnestic and amnestic subtypes, respectively [27]. Hypofrontality in our TC-99 m-ECD SPECT observations also provides an interesting parallel to another cohort study, in which predominant post-central cerebral blood flow decrement with better preserved central and prefrontal flow value is described among Cbl-deficient patients with heterogenous dementia subtypes and superimposed delirium [28]. As all of our patients with cognitive complaints are free of delirium and/or of psychotropic medications before TC-99 m-ECD SPECT evaluations, the linkage between functional neuroimaging and the cognitive deficits relevant to $\mathrm{Cbl}$ deficiency would be more straightforward. Still, doubt on co-existing neurodegenerative disease in our cohort might exist. We regard Cbl deficiency as the main causes of cognitive impairment in the current study based onto their structural images are nearly normal. While hypoperfusion of TC-99 m-ECD SPECT is pronounced, the volume of brain parenchyma remains fully preserved. Moreover, the signal reversal of follow-up Tc-99 m-ECD SPECT after Cbl replacement therapy supports the linkage between low $\mathrm{Cbl}$ status and cerebral metabolic changes. The extent and degree of signal reversal of TC-99 m-ECD SPECT, in line with cognitive changes, serves as useful internal control to validate our hypothesis.

There are several lines of mechanisms explaining symptoms attributed to Cbl deficiency. First is the derangement in the monoamine neurotransmitter, as both $\mathrm{Cbl}$ and folic acid are prerequisites for its production [4]. Second is impaired DNA synthesis by reducing availability of tetrahydrofolate, the essential substance maintaining cellular integrity [29]. Third is damages of vessels and myelin secondary to acumination of homocysteine and methylmalonic acid [30]. Given with nearly-normal conventional MRI images in this study, we therefore assume that neurotransmitter derangement and damage of neuronal integrity contribute to the hypometabolism of fronto-temporal regions on TC-99 m-ECD SPECT, as regional cerebral blood flow has been regarded to reflect synaptic activities [31]. Our hypothesis is also in line with previous animal experiments, issuing $\mathrm{Cbl}$ deficiency would impair cerebral glucose metabolism in cortical as well as subcortical areas of the brain [32].

It's also worth to point out that the metabolic derangement among Cbl-deficient patients predates obvious changes of structural neuroimaging, even among young patients. In our cohort, there were two patients, aged 29 and 41, presented psychiatric symptoms and relevant hypoperfusion restricted within temporal regions and deep nuclei. These two patients, whose ages are much younger than those of patients with neurodegenerative disease, address evidences supporting negative impact of $\mathrm{Cbl}$ deficiency toward central nervous system. Some may speculate whether TC-99 m-ECD SPECT findings are the epiphenomenon related to endogenous mood disorders rather than the results relevant to $\mathrm{Cbl}$ deficiency. Nonetheless, their low $\mathrm{Cbl}$ level raises the plausible explanation to their unusual presentations, which is distinct from typical mood disorders. Moreover, half of our patients with follow-up neuropsychiatric tests show remarkable improvement after 3 months of Cbl supplement therapy. The robust findings mirror the causality between cognitive impairment and $\mathrm{Cbl}$ deficiency. Although poor responders to $\mathrm{Cbl}$ supplement exist, such findings don't preclude the negative impact of low $\mathrm{Cbl}$ state, as normalization of serum Cbl level doesn't guarantee complete restoration of cellular reserve and utilization process [1].

There are several limitations in our study. First, the study was limited in its cross-sectional study design as well as small sample size. Generalization of the results warrants future prospective studies of greater case number. Comparison of SPECT findings between groups with similar cognitive achievement but different $\mathrm{Cbl}$ level would also of high research value to document linkage between $\mathrm{Cbl}$ level and SPECT presentations. Second, as excluding co-existing neurodegenerative disease has always been a great challenge in the studies related to $\mathrm{Cbl}$ deficiency, we had integrated brain MRI into diagnostic consideration. We regard the possibility of co-existing Alzheimer's disease in our cohort is limited, as our patients have no medial temporal lobe atrophy, the pathological change typically observed in Alzheimer's disease or amnestic type of MCI. The signal reversal after $\mathrm{Cbl}$ replacement also served as evidences to corroborate that the underlying pathogenesis in our cohort is related to Cbl deficiency. Third, the study of other serology markers related to $\mathrm{Cbl}$ was not comprehensive in our study. Due to the fact that serum methylmalonic acid and homocysteine elevates earlier than the serum $\mathrm{Cbl}$ decrement [33] and holotranscobalamin, the biologically active $\mathrm{Cbl}$, correlates with $\mathrm{Cbl}$ status in a more sensitive manner [34,35], application of these serum markers in the future studies may provide further information fundamental for changes of cerebral metabolism. Last, although our TC-99 m-ECD SPECT evaluations provided convincing evidences representing functional derangement within selected regions, future studies, preferably analyzing segregated anatomical regions, may 
be more straightforward underpinning cerebral metabolic changes attributed to $\mathrm{Cbl}$ deficiency.

\section{Conclusion}

The retrospective review of our TC-99 m-ECD SPECT observations provides pivotal information of neurobiological changes within basal ganglia and fronto-temporal regions in conjunction with disease severity among patients with $\mathrm{Cbl}$ deficiency. Hypoperfusion within basal ganglia in addition to temporal regions may be seen in the earlier state of Cbl deficiency, when cognition remains intact but psychiatric symptoms predominate. Hypoperfusion within basal ganglia in association with frontal regions appears when cognitive problems, mostly dysexecutive syndrome, are manifested. Symmetric hypofrontality in association with dysexcutive syndrome serves as a distinguishing feature of non-amnestic $\mathrm{MCI}$ attributed to $\mathrm{Cbl}$ deficiency, in contrast to amnestic MCI or Alzheimer's disease. Concordant with TC-99 mECD SPECT findings, the psychiatric symptoms and dysexcutive syndrome undergird impaired limbic and dorsolateral prefrontal circuits originated from basal ganglia respectively. As prompt supplement reverses cognitive deficits among certain portion of patients with $\mathrm{Cbl}$ deficiency, judicious evaluation of $\mathrm{Cbl}$ status should be highlighted among patients with non-amnestic MCI, especially in the context of poor nutrition intake and/or malabsorption.

\section{Abbreviations}

CASI: cognitive abilities screening instrument; Cbl: cobalamin; CDR: clinical dementia Rating; MCl: mild cognitive impairment; MMSE: mini-mental state examination; MRI: magnetic resonance imaging; NPI: neuropsychiatric inventory; Tc-99 m-ECD SPECT: Tc-99 m ethyl cysteinate dimer single-photon emission computed tomography.

\section{Competing interests}

The authors have no financial or non-financial disclosures to make or potential conflicts of interest to report in relation to this investigator-led study.

\section{Authors' contributions}

MCT drafted the manuscript for content, participated study concept and carried out data interpretation. CPL: interpreted MRI, participated study concept and revised the manuscript. CYC: interpreted TC-99 m-ECD SPECT scan, carried out data interpretation and revised the manuscript. CFH: interpreted neuropsychological tests and revised the manuscript. All authors read and approved the final manuscript.

\section{Authors' information}

MCT and CFH: Attending staffs and specialists of higher cortical function in Department of Neurology. Lecturers of School of Medicine, Tzu Chi University, Hualien, Taiwan.

CPL: Attending staff and specialist of neuroradiology in Department of Radiology. Assistant Professor of School of Medicine, Tzu Chi University, Hualien, Taiwan.

CYC: Attending staff and specialist of neuroradiology in Department of Nuclear Medicine. Lecturer of School of Medicine, Tzu Chi University, Hualien, Taiwan.

\section{Acknowledgements}

The authors thank the patients and their caregivers for their time and commitment to this research. We also appreciate Mr. Tien-Hsin Chang, who assisted SPECT imaging processing.

\section{Author details}

${ }^{1}$ Department of Neurology, Taichung Tzu Chi Hospital, Buddhist Tzu Chi Medical Foundation, Taichung, Taiwan. ${ }^{2}$ Department of Radiology, Taichung Tzu Chi Hospital, Buddhist Tzu Chi Foundation, Taichung, Taiwan. ${ }^{3}$ Department of Nuclear Medicine, Taichung Tzu Chi Hospital, Buddhist Tzu Chi Medical Foundation, Taichung, Taiwan. ${ }^{4}$ School of Medicine, Tzu Chi University, Hualien, Taiwan. ${ }^{5}$ Graduate Institute of Medical Imaging and Radiological Sciences, Central Taiwan, University of Science and Technology, Taichung, Taiwan.

Received: 8 June 2015 Accepted: 19 November 2015

Published online: 03 December 2015

\section{References}

1. Lachner C, Steinle NI, Regenold WT. The neuropsychiatry of vitamin B12 deficiency in elderly patients. J Neuropsychiatr Clin Neurosci. 2012;24:5-15.

2. Knopman DS, DeKosky ST, Cummings JL, Chui H, Corey-Bloom J, Relkin N, et al. Practice parameter: diagnosis of dementia (an evidence-based review): report of the quality standards subcommittee of the american academy of neurology. Neurology. 2001;56:1143-53.

3. Petersen RC, Doody R, Kurz A, Mohs RC, Morris JC, Rabins PV, et al. Current concepts in mild cognitive impairment. Arch Neurol. 2001;58:1985-92.

4. Hutto BR. Folate and cobalamin in psychiatric illness. Compr Psychiatry. 1997;38:305-14.

5. Vogiatzoglou A, Refsum H, Johnston C, Smith SM, Bradley KM, de Jager C, et al. Vitamin B12 status and rate of brain volume loss in communitydwelling elderly. Neurology. 2008;71:826-32.

6. Tangney CC, Aggarwal NT, Li H, Wilson RS, Decarli C, Evans DA, et al. Vitamin B12, cognition, and brain MRI measures: A cross-sectional examination. Neurology. 2011;77:1276-82.

7. Blundo C, Marin D, Ricci M. Vitamin B12 deficiency associated with symptoms of frontotemporal dementia. Neurol Sci. 2011:32:101-5.

8. Akdal G, Yener GG, Kurt P. Treatment responsive executive and behavioral dysfunction associated with Vitamin B12 deficiency. Neurocase. 2008;14:147-50.

9. Stott DJ, Maclntosh G, Lowe GD, Rumley A, McMahon AD, Langhorne P, et al. Randomized controlled trial of homocysteine-lowering vitamin treatment in elderly patients with vascular disease. Am J Clin Nutr. 2005;82:1320-6.

10. Tanaka F, Vines D, Tsuchida T, Freedman M, Ichise M. Normal patterns on ${ }^{99 m}$ Tc-ECD brain SPECT scans in adults. J Nucl Med. 2000;41:1456-61.

11. Folstein MF, Folstein SE, McHugh PR. "Mini-mental state". A practical method for grading the cognitive state of patients for the clinician. J Psychiatr Res. 1975;12:189-98.

12. Teng EL, Hasegawa K, Homma A, Imai Y, Larson E, Graves A, et al. The Cognitive Abilities Screening Instrument (CASI): A practical test for cross-cultural epidemiological studies of dementia. Int Psychogeriatr. 1994;6:45-56.

13. Morris JC. The Clinical Dementia Rating (CDR): current version and scoring rules. Neurology. 1993:43:2412-4.

14. Cummings JL, Mega M, Gray K, Rosenberg-Thompson S, Carusi DA, Gornbein J. The Neuropsychiatric Inventory: comprehensive assessment of psychopathology in dementia. Neurology. 1994:44:2308-14.

15. Lin KN, Wang PN, Liu HC, Teng EL. Cognitive Abilities Screening Instrument, Chinese version 2.0 (CASI C-2.0): Administration and clinical application. Acta Neurol Taiwanica. 2012;21:180-9.

16. Moore TL, Schettler SP, Killiany RJ, Rosene DL, Moss MB. Effects on executive function following damage to the prefrontal cortex in the rhesus monkey (Macaca mulatta). Behav Neurosci. 2009;123:231-41.

17. Duncan J, Owen AM. Common regions of the human frontal lobe recruited by diverse cognitive demands. Trends Neurosci. 2000;23:475-83.

18. Amen DG, Trujillo M, Newberg A, Willeumier K, Tarzwell R, Wu JC, et al. Brain SPECT imaging in complex psychiatric cases: an evidence-based, underutilized tool. Open Neuroimag J. 2011:5:40-8.

19. Shallice $T$, Burgess $P$. The domain of supervisory processes and temporal organization of behaviour. Philos Trans R Soc Lond B Biol Sci. 1996;351:1405-11. 
20. Miller EK, Cohen JD. An integrative theory of prefrontal cortex function. Annu Rev Neurosci. 2001;24:167-202.

21. Banich MT. Executive function: The search for an integrated account. Curr Dir Psychol Sci. 2009;18:89-94.

22. Ring HA, Serra-Mestres J. Advance in neuropsychiatry Neuropsychiatry of the basal ganglia. J Neurol Neurosurg Psychiatry. 2002;72:12-21.

23. Squire LR, Alvarez P. Retrograde amnesia and memory consolidation: a neurobiological perspective. Curr Opin Neurobiol. 1995;5:169-77.

24. Farah MJ, Gillihan SJ. The puzzle of neuroimaging and psychiatric diagnosis: technology and nosology in an evolving discipline. AJOB Neurosci. 2012:3:1-11.

25. Borroni B, Anchisi D, Paghera B, Vicini B, Kerrouche N, Garibotto V, et al. Combined 99mTc-ECD SPECT and neuropsychological studies in $\mathrm{MCl}$ for the assessment of conversion to AD. Neurobiol Aging. 2006;27:24-31.

26. Bennett DA, Wilson RS, Schneider JA, Evans DA, Beckett LA, Aggarwal NT, et al. Natural history of mild cognitive impairment in older persons. Neurology. 2002;59:198-205.

27. Nobili F, Frisoni GB, Portet F, Verhey F, Rodriguez G, Caroli A, et al. Brain SPECT in subtypes of mild cognitive impairment. Findings from the DESCRIPA multicenter study. J Neurol. 2008;255:1344-53.

28. Nilsson K, Warkentin S, Hultberg B, Fäldt R, Gustafson L. Treatment of cobalamin deficiency in dementia, evaluated clinically and with cerebral blood flow measurements. Aging (Milano). 2000;12:199-207.

29. Banerjee RV, Matthews RG. Cobalamin-dependent methionine synthase. FASEB J. 1990;4:1450-9

30. Scalabrino $\mathrm{G}$. The multi-faceted basis of vitamin B12 (cobalamin) neurotrophism in adult central nervous system: Lessons learned from its deficiency. Prog Neurobiol. 2009;88:203-20.

31. Jueptner M, Weiller C. Review: does measurement of regional cerebral blood flow reflect synaptic activity? Implications for PET and fMRI. Neuroimage. 1995;2:148-56.

32. Hakim AM, Cooper BA, Rosenblatt DS, Pappius HM. Local cerebral glucose utilization in two models of B12 deficiency. J Neurochem. 1983;40:1155-60.

33. Lindenbaum J, Savage DG, Stabler SP, Allen RH. Diagnosis of cobalamin deficiency: II. Relative sensitivities of serum cobalamin, methylmalonic acid and total homocysteine concentrations. Am J Hematol. 1990;34:99-107.

34. Hvas AM, Nexo E. Holotranscobalamin-a first choice assay for diagnosing early vitamin B deficiency? J Intern Med. 2005;257:289-98.

35. Hooshmand B, Solomon A, Kareholt I, Rusanen M, Hanninen T, Leiviska J, et al. Associations between serum homocysteine, holotranscobalamin, folate and cognition in the elderly: a longitudinal study. J Intern Med. 2012:271:204-12.

\section{Submit your next manuscript to BioMed Central and we will help you at every step:}

- We accept pre-submission inquiries

- Our selector tool helps you to find the most relevant journal

- We provide round the clock customer support

- Convenient online submission

- Thorough peer review

- Inclusion in PubMed and all major indexing services

- Maximum visibility for your research 